\title{
Contexto, gestores y caracterización de las Resource and Description Access (RDA)
}

Resource and Description Access (RDA): context, managers and characterization

\author{
Ignacio López GUILLAMóN \\ Servicio de Archivos, Bibliotecas y Documentación, Universidad de Extremadura (España), ignaciolg@unex.es
}

\begin{abstract}
Resumen
Se expone una caracterización de las RDA, que es el código internacional de catalogación en proceso de implantación en las más importantes bibliotecas y redes bibliotecarias de los países de lengua inglesa y el modelo normativo de la catalogación bibliográfica de la información digital. Se muestra el valor de la tecnología digital aplicada a los procesos de representar y comunicar los datos bibliográficos y las relaciones de indización en la difusión del conocimiento presente. Se presenta cómo los cambios sucedidos a partir de las TICs han transformado la catalogación bibliográfica en elaboración de metadatos y en la primera configuración de las RDA. Se resaltan las actuaciones impulsadas por la IFLA, el Joint Steering Committee for Revision of AACR (JSCR of AACR) 0 The European RDA Interest Group (EURIG).
\end{abstract}

Palabras clave: Resource and Description Access (RDA). Descripción bibliográfica. Catalogación bibliográfica. Metadatos.

\section{Objetivos, metodología y plan de trabajo}

El presente ensayo muestra las claves de los cambios producidos en los presupuestos especulativos y empíricos de la catalogación bibliográfica de nuestros días. Por un lado, se presenta la transformación obrada en el objeto de la catalogación: los recursos informativos. Aborda, en segundo lugar, cómo el producto de la catalogación y la elaboración de metadatos, por efecto de las Tecnologías de la Información y la Comunicación (TICs), ofrecen unos resultados convergentes.

En tercer lugar, siendo la RDA: Descripción y acceso a recursos (2010) la propuesta más innovadora en materia de catalogación del presente, se ha estimado necesario presentar qué instituciones hay tras ella, cómo se ha generado y una caracterización básica de la misma, en el afán de contribuir a su difusión entre la comunidad profesional de catalogadores.

La RDA se define como conjunto de directrices e instrucciones para describir y acceder a los

\begin{abstract}
A characterization of RDA is presented, which is the international cataloging code currently being implemented in major libraries and library networks in the English language countries and the normative model of bibliographic cataloging digital information. The aim is obtaining a better understanding of the context, agents and scope of the RDA, that show the value of digital technology applied to the processes of representing and communicating bibliographic data and indexing relations. The article highlights the actions promoted by IFLA, the Joint Steering Committee for Revision of AACR (JSCR of AACR) or The European RDA Interest Group (EURIG).
\end{abstract}

Keywords: Resource and Description Access RDA). Bibliographic description. Cataloguing.

recursos, analógicos y digitales, a partir de registros bibliográficos y de autoridad, independientemente de cómo se codifique la información (ISBD, MARC21, Dublin Core o MODS).

En este ensayo se ha seguido un método de investigación descriptivo, acorde con la naturaleza expositiva y explicativa del mismo. El análisis cualitativo de la más reciente innovación producida en el ámbito de la catalogación bibliográfica se ha planteado desde el criticismo racionalista de K. Popper. El método sistémico nos ha permitido comprender mejor los contextos teóricos y prácticos conducentes a las RDA. Igualmente, la teoría de modelos de Thomas S. Kuhn ha servido para entender y explicar el agotamiento del modelo de la catalogación automatizada y cómo éste deja paso al nuevo marco teórico y empírico de la catalogación digital.

Las referencias que han servido para elaborar este ensayo han sido fuentes primarias. Con ellas no se agota el tema de estudio, dado el fin de presentar un enfoque conciliador entre la tradición catalográfica y la metamorfosis que las 
TICs han generado en la nueva formulación de la RDA para describir y acceder a los recursos informativos.

El esfuerzo realizado desde los años 90 por revisar la reglamentación catalográfica vigente - así como los análisis que simultáneamente se han llevado a cabo sobre la operación de catalogar, y su conceptualización, hasta redactar los FRBR (1997), FRAD (2008) y otros- marcan, de hecho, el fin de una etapa caracterizada por la información analógica y el comienzo de la información digital.

Entre uno y otro modelo el nexo es la catalogación bibliográfica elevada a la categoría de disciplina conceptual y empírica, cuyo objeto es la comunicación de los saberes en la sociedad, independientemente del soporte material, el medio de acceso a la información, su codificación lingüística y cualesquiera otros accidentes materiales, culturales y técnicos.

Un nuevo marco tecnológico afecta a la presentación y comunicación de los recursos informativos e, igualmente, al proceso de difusión y acceso del conocimiento en el presente. La comunicación del saber ha encontrado en la trama de la descripción de objetos metadatos (MODS), en la de los datos de autoridad (MADS) y en la codificación y transmisión de estos (METS) un nuevo escenario y actores en el proceso del trabajo científico: el acceso y recuperación de los conocimientos.

En la transición al siglo XXI, las TICs han resaltado el agotamiento de un modelo catalográfico, cuya elaboración teórica data del siglo XIX y cuyas actualizaciones durante el siglo $X X$ resultan ya inoperantes ante las posibilidades comunicativas e informativas de los nuevos recursos informativos del presente. La tensión teórica y práctica producida por las TICs en la operación de catalogar ha sido analizada por los expertos de las dos últimas décadas. Éstos han resaltado el valor de las funcionalidades esenciales de la misma frente a las puestas al día de épocas anteriores. Con ello, se ha reforzado el valor especulativo y empírico que la catalogación bibliográfica posee en la comunicación de los conocimientos en cualquier etapa histórica.

Los Principios internacionales de catalogación (2009) expresan las funciones y objetos de la catalogación y de los catálogos. Y la RDA, Descripción y acceso a recursos (2010) se propone como apuesta mundial por establecer una codificación internacional acorde con la realidad tecnológica digital, que afecta a la materialidad de los recursos informativos, a su codificación y a su comunicación.
La metamorfosis de la catalogación bibliográfica, por efecto de la tecnología digital, está motivada por los cambios operados en el sujeto libro a los que se aplica; en los procedimientos de representación y transmisión de los datos descriptivos e indiciales; $y$, también, en la naturalización del acceso y recuperación de la información en la sociedad como factor de aprendizaje y de renovación constante de los conocimientos.

La catalogación bibliográfica ha devenido en la disciplina teórica y práctica que sistematiza los conocimientos y los relanza a la sociedad a partir de su representación fiel y unívoca. Ahora bien, la fijación de los datos bibliográficos y sus relaciones indiciales deben regirse por principios y formalidades orientadas a la eficiencia de la comunicación del saber, en su doble aspecto de difundir, de una parte; y de acceder a la información, de otra. Esta funcionalidad recíproca es la clave conceptual y empírica de la RDA.

\section{Actualidad de los recursos digitales}

\subsection{Universo bibliográfico}

Universo bibliográfico es un concepto que se ha creado en el marco de las distintas reuniones de la IFLA, celebradas entre 2003 y 2007 en cinco ciudades estratégicas respecto de la tradición catalográfica, para designar aquello que es común a las colecciones que se integran en bibliotecas, archivos, museos y otras comunidades de información. Es una expresión llamada a tener éxito por el hecho de aunar el fenómeno de la información, independientemente de su enunciado más particular. Hace, pues referencia, al complejo sistema de expresión de los conocimientos en cualquier soporte para su comunicación más general. El calificativo bibliográfico está en relación con la forma de comunicación expresada por un saber y el sentido humano por el que se pretende que sea éste percibido.

El universo bibliográfico engloba los testimonios del saber que se sistematizan y ofrecen a la sociedad en distintas comunidades de información, como son las bibliotecas, archivos y museos. Se trata de integrar la información de naturaleza estrictamente libraría con la derivada de otras realidades culturales, que, como el libro, constituyen otras manifestaciones del saber y que emplean los metadatos en su codificación y difusión.

En este universo bibliográfico importa más la información precisa para la descripción de un recurso, sea éste analógico, o digital, que la forma de su presentación. No obstante, conscientes de estar en una etapa de transición, los 
estándares ISBD, así como la codificación en MARC21, son estructuras para la codificación y transferencia de la información bibliográfica valoradas en la nueva etapa que se abre para la catalogación a partir de RDA.

Ésta se presenta como una normativa acorde con la descripción y acceso a recursos digitales, toda vez que las bases de datos bibliográficas actuales son exponentes de la tecnología digital, como también lo son bastantes medios de trabajo que ayudan en el quehacer de los profesionales de la catalogación. Oliver Chris resalta cómo los cambios operados entre 1960 y 2000 han sido determinantes para transformar la praxis de la catalogación (Chris, 2010, p. 5-9).

\subsection{Afianzamiento de los recursos digitales}

Este universo bibliográfico, en los últimos años, es digital. Los agentes que participan en la realidad digital son los autores, editores, distribuidores, libreros y gestores de la información. El International Digital Publishing Forum informa que más del $93 \%$ de toda la información que se produce se hace en formato digital. Según esta misma entidad, el libro digital se afianza sobre el modelo electrónico de documento PDF.

Las monografías comenzaron a difundirse en versión digital junto con los recursos continuados. Sin embargo, es a partir de 2007, con el desarrollo masivo de los lectores electrónicos de pantalla de tinta electrónica (e-readers) cuando los libros digitales resultan tan atractivos como los impresos. La expansión de Google Book y de Open Content Alliance, en el ámbito internacional, son exponentes de esta misma apuesta por la difusión digital de las obras de naturaleza monográfica. Para el caso de España, habría que citar TodoeBook y Libranda. Sobre estos pueden analizarse los trabajos del Observatorio de la Lectura y el Libro (El libro..., 2010).

La posibilidad de que el e-readers disponga de papel y tinta electrónicas recuerdan los esfuerzos de diseño hechos para que los incunables imitasen en todo al manuscrito. Se trata de dispositivos compactos, que remedan al formato libro, con una pantalla entre 5 y 10 pulgadas, que carece de iluminación propia, es de bajo consumo y posee un contraste acusado, que permite leer bajo iluminación solar.

Su batería permite almacenar en torno a las 10.000 páginas, son cómodos de llevar, disponen de doble memoria (interna y para tarjetas SD). Algunos ofrecen un teclado QWERTY y con ello se multiplican sus funcionalidades. En otros casos, una pantalla táctil cubre aplicaciones como subrayar, escribir notas, etc. Cuentan, también, con la posibilidad de conectar a Internet por Wi-fi o 3G, muy extendida entre los teléfonos móviles.

Para una mejor visualización de Internet, se trabaja en la consecución de unas pantallas con doble interfaz, una con papel y tinta electrónicas para la lectura de obras; y otra retroiluminada para el acceso a Internet. Algo pendiente de resolución final son las conversiones de los distintos formatos en los que se presentan los recursos electrónicos (RTF, PDF, ePUB, FB2, MOBI...). A tal fin pueden servir Calibre, PDFCreator, ePub2 Go, Coversor grammata, Sigil, etc. Más información puede encontrarse en el trabajo conjunto de José Antonio Cordón García, Julio Alonso Arévalo y Helena Martín Rodero (2010, p. 53-80).

\subsection{Incidencia de los recursos digitales en la biblioteca}

Presentados los dispositivos y algunas herramientas técnicas se pasa a exponer que la demanda social y el marketing empresarial han llevado esas realidades a la biblioteca anglosajona y se está experimentando en el resto de ámbitos idiomáticos. Aun siendo cierto que se está en los inicios de ofertar las obras en versión impresa y en versión digital, no lo es menos que esa oferta es ya una realidad y de que se está evaluando sus costes y potencialidades de servicio.

Las compras selectivas, o por paquetes, a perpetuidad o a partir de licencias renovables; el acceso restringido, el acceso libre, sistemas de gestión de derechos digitales (DRM) (Alonso y Cordón, 2010a), etc., son aspectos que en breve van a determinar el éxito de la edición digital. Una visión más completa se encuentra en El libro electrónico en la biblioteca digital: modelos de negocio y política de precios (2010).

El incremento paulatino de las obras electrónicas debe integrarse en los catálogos electrónicos de la biblioteca, ya sea en el mismo sistema de gestión de las colecciones impresas o en sus distintos soportes vigentes hasta el presente, bien en plataformas de los editores. Sin duda la experiencia habida con la andadura seguida por las publicaciones periódicas servirá en el desarrollo de la edición digital de las monografías (Encuesta..., 2009). Pueden suceder soluciones mixtas, toda vez que los editores ofertan las obras digitales con catalogación realizada en MARC21.

Con los dispositivos de lectura e-books se constata que las bibliotecas ofrecen en préstamo ereaders, tal y como sucede con otra categoría 
de equipos técnicos, como son los ordenadores portátiles. En función del contrato fijado con la editorial, si el libro digital se ofrece por wi-fi, la consulta es multiusuario y multilocal. Sólo es preciso identificarse como usuario de tal biblioteca. Pero en otros supuestos, el e-reader permite el acceso libre por un tiempo determinado a la lectura de una obra digital, que está gestionada por un DRM; y, transcurrido el tiempo previsto, desaparece del e-readers para pasar a otro usuario.

Con estos servicios, la biblioteca, entendida como espacio público, se transforma en un lugar de encuentro para usuarios de intereses comunes. Por lo general, el web de estas bibliotecas ofrece localizadores de las obras deseadas. Es bastante evidente el paralelismo que se da entre los servicios presenciales y los servicios en línea.

\subsection{Caracterización del recurso digital}

Todo apunta a la transformación del libro impreso en libro digital. Se trata de una asimilación con ventajas en acercar de inmediato a los usuarios interesados a las obras en cualquier parte del mundo; $y$, también, en agilizar las relaciones entre el autor de la obra, el editor y el usuario final.

Una consecuencia de esta activación del proceso de comunicar los conocimientos o las creaciones intelectuales y artísticas puede revertir e una disminución de los costes y, consecuentemente, en un mayor desarrollo y más rápida difusión e implantación (Alonso y Cordón, 2010b, p. 58-68). Sobre la gestión de las licencias, los usos y los derechos de autor del libro digital puede consultarse el trabajo de José A. Cordón García y de Julio Alonso Arévalo presentado al $V$ Congreso Nacional de Bibliotecas Públicas, Gijón, 2010.

El recurso digital se caracteriza por: (1) fácil movilidad, lo cual está en relación directa con su peso, volumen y autonomía de uso; (2) interoperbilidad de los contenidos que pueden vehicular de un soporte a otro, en algunos casos; (3) actualización sistemática del contenido; y (4) posibilidad de interactuar entre los responsables de los contenidos y la comunidad a la que se dirige.

Un informe sobre el libro digital en Francia indica que el libro digital ha tenido un mayor desarrollo en la biblioteca digital, frente a lo sucedido con los recursos con contenidos de música o de imágenes en movimiento (Patiño, 2009, p. 4-5). Esto se explica por que el paso de la obra impresa a la obra digital ha tenido lugar en mu- chos casos por la edición en CD-ROM y DVDROM de múltiples contenidos científico-técnicos y especializados. Al mismo tiempo la gestión de licencias, explotación de funcionalidades y aspectos legales de los derechos de autor de tales recursos se desarrollaron gradualmente.

Es un hecho que la información digital está extendida entre la comunidad académica, en los sectores vinculados con el derecho y la jurisprudencia, en el ámbito de la educación primaria y secundaria y entre los sectores sociales cuya actividad económica se relaciona con la innovación y el turismo.

En cualquier caso, el recurso digital exige que los editores y distribuidores asuman el control de las obras digitales y su comercialización, a partir de reglamentaciones legales que garanticen la libre disposición de los conocimientos y el correspondiente respeto legal de los derechos de autor.

Igualmente, la visibilidad de tales recursos resulta esencial para su libre comunicación. Es necesario presentar la noticia de tales recursos digitales de forma que, como sucede con el libro, sean conocidos los responsables, los títulos, los editores y cualquier otra circunstancia que condicione el acceso y recuperación formal de tales recursos. Es esencial regular todos los niveles la gestión digital de los derechos de autor (DRM) para sentar las bases de un sólido y extenso desarrollo de la edición digital (Cordón, Gómez y Alonso, 2010).

El desarrollo progresivo de la tecnología digital se acusa en la comunicación de los conocimientos y afecta a cualquier ámbito profesional. El potencial de transformación de tal tecnología es valorado por las instancias gubernamentales con medidas de apoyo y promoción. Un escenario privilegiado para la generación y transmisión de los conocimientos sobre y desde la tecnología digital es la enseñanza superior. Por ello, en el ámbito europeo se pretende uniformar tal nivel de enseñanza a fin de que una cualificación profesional homogénea, instrumentalizada por la tecnología digital, sirva para elevar las cotas de cohesión social y económica y, en definitiva, de desarrollo.

\section{Catalogación y metadatos}

\subsection{Génesis de los metadatos}

El pórtico sobre la asunción de la realidad digital aplicada a la difusión y acceso a los saberes conlleva a exponer el concepto de metadatos; así como algunas de las reflexiones sobre las coincidencias y divergencias existentes con la 
operación de catalogar y con el registro bibliográfico y su desenlace final, en la consideración de Barbara B. Tillett.

Priscilla Caplan (1995, p. 19-23) señalaba hace años cómo los metadatos eran datos sobre datos y poseían similitudes con los registros bibliográficos resultantes de la operación de catalogar. Por su parte, Tim Berners-Lee (1997, revisado en 2009) apunta las diferencias entre ambas realidades al señalar que los metadatos son información inteligible por el ordenador, que se aplican a recursos web u otros productos.

Stuart Weibel y Juha Hakela (1998) proponen que el autor del documento puede ser quien elabore el metadato. Por su parte, Karl Lagoze (1997) resalta que los metadatos facilitan el encuentro de los recursos en un medio digital. Hay más elementos diferenciadores, como son el que los metadatos se conciben desde la óptica de servir a los usuarios finales para obtener y relacionar los recursos digitales por Internet.

Ingrid Hsieh-Yee (1997) estima que los registros bibliográficos y los metadatos sirven por igual a la organización de la información, por cuanto seleccionan, describen y ofrecen puntos de acceso autorizados, análisis de su contenido y su localización, pese a que el registro bibliográfico se hace sobre ciertas categorías de documentos y los metadatos se hace sobre los recursos web. La coincidencia parcial ha llevado a autores como Stefan Gradmann (1998), Grete Pasch (2001), Ania Torres (2006), entre otros, a establecer paralelismos entre ambas realizaciones.

\subsection{Caracterización de los metadatos}

Los metadatos constan de distintos datos estructurados sobre un recurso digital. Estos se integran en el propio recurso ya sea a partir de una etiqueta con la dirección URL en una base de datos, ya sea a partir de un encabezamiento SGML, de forma tal que es recuperable por un buscador.

Funcionalmente, los datos informativos que identifican y sirven de acceso a un recurso a partir de un metadato pueden, en buena lógica, ser coincidentes con los datos que conforman un registro bibliográfico. Pero no son iguales desde un punto de vista más técnico, pues los datos del registro bibliográfico son, además, exponentes de una normalización y de una diferenciación formalista que les lleva a ofrecer una cohesión y consistencia informativa en cualquier prontuario bibliográfico que los metadatos carecen (Rusch-Feja, 1998). Los metadatos responden al fin de intercambiar información entre los buscadores digitales, agilizando el acceso y recuperación de los recursos digitales.

Ernest Abadal (1994, p. 371-391) señala cómo la tecnología digital de la información y de la comunicación ha dado naturaleza a los metadatos, cómo éstos sirven en este entorno tecnológico y cómo una cierta normalización y formalización contribuiría a que fueran más precisos al ofrecer los resultados informativos de los metabuscadores.

Un trabajo colectivo de algunos bibliotecarios noruegos presenta en 2004 (Van Nuys, Albertsen, Pedersen y Stenstad, 2004) los rasgos distintivos de los metadatos y la complejidad de concretar los límites de un recurso digital en Internet, a saber: (1) interoperabilidad semántica con MARC; (2) requisitos mínimos funcionales; (3) posibilidad de conversión a distintos formatos de codificación de datos entre MARC21 y MODS, ONIX, Dublin Core; (4) posibilidad de conversión a XML; (5) extensibilidad que permita definir nuevos elementos futuros; y (6) disponer de un marco de referencia común, que permita la identificación y conversión de elementos semejantes a efectos técnicos, de derechos o gestión.

Para ampliar el concepto, funcionalidades y desarrollo del metadato pueden consultarse fuentes como UKOLN, PADI: preservation to digital information o la misma IFLA.

El estudio sobre las publicaciones de Eva María Méndez Rodríguez (2002-2010) acerca de los metadatos (más de ocho trabajos) es exponente del cambio conceptual y tecnológico obrado en éstos. Hasta tal punto son importantes esos cambios que Barbara B. Tillett, en 2010, identifica los resultados de la operación de catalogar a la elaboración de metadatos. Para esta autora, desde la universalización del modelo FRBR y FRAD el resultado de aplicar una serie de estándares a la catalogación bibliográfica y la elaboración de metadatos es una misma realidad (Tillett, 2009, p. 16, 23-24, 29).

La formulación de FRBR ha sido un revulsivo tal en los procedimientos de configurar los datos sobre los documentos, analógicos o digitales, que los expertos en catalogación y en elaborar metadatos han sumado esfuerzos, de modo que OCLC, AustLit o VTLS, entre otros, han asumido este modelo en sus procesos de identificación y organización de los datos de los recursos informativos.

Dublin Core Abstract Model ha asumido como propio el paradigma FRBR. Se estima que será una gran ventaja a efectos prácticos de trabajo el establecer relaciones entre las entidades 
obra, manifestación, expresión y ejemplar. La tecnología web aplicada a catálogos y a todo tipo de recursos permite el acceso y recuperación de cualquier recurso informativo en línea, inclusive las monografías, como ha quedado patente en el primer apartado de este ensayo.

Igualmente, la mundialización de la tecnología digital guarda una estrecha relación con el concepto de universo bibliográfico, que a diario interactúa cada vez más con la generación social del presente. Siendo éste el escenario de fondo, se deduce que la formación permanente de los profesionales debe activarse en esta línea de trabajo.

\subsection{Implicaciones de los metadatos en la biblioteca}

Roy Tennant (2009) en un sugerente trabajo expone que el objetivo de la descripción bibliográfica se concreta en permitir que personas independientes y autosuficientes encuentren lo que desean de una biblioteca, esencialmente, digital. Esto obliga a reducir la operación y mecánica de búsqueda de la información a la intuición del usuario, prescindiendo de las secuencias de tipo bibliográfico por otras genéricas, tales como lugar, fecha, género, tema... Hoy cualquier OPAC obliga a buscar por dichas categorías de datos y se puede limitar la búsqueda indicando fechas, tipos de documentos...

En tal sentido trabajan desde hace unos años los principales agentes bibliotecarios del mundo occidental, encabezados por las grandes bibliotecas y redes bibliotecarias anglosajonas. Los metadatos $-\mathrm{y}$ un registro bibliográfico es un conjunto de metadatos- son determinantes en los procesos de recuperación de la información digital, ya sea para distinguir dos muy similares, ya sea como filtro de nivel intelectual... Se impone enriquecer y gestionar más eficientemente la descripción bibliográfica si se desea mejorar la posterior recuperación de la información.

Cita R. Tennant como centros de vanguardia a OCLC y su producto más extendido, el WorldCat, o el Proyecto Europeana. Hoy se pide a los editores que aporten la información de sus libros en el formato ONIX XML, que OCLC pasa a MARC para incluirlos en WorldCat. El formato ONIX ofrece información adicional sobre el autor o el título del recurso, que puede interesar al usuario.

Otra iniciativa es enriquecer los registros con el índice de la obra. Muy conocido es, igualmente, el Bibliographic Enrichment Advisory Team (BEAT), de la Library of Congress. La interacción con los usuarios es también un reto de acción inminente. Esto es posible a partir del uso de un interfaz de programación de aplicaciones (API), a través de Open Archives Initiative Protocol for Metadata Harvesting (OAI-PMH) (The Open..., 2008) y, también, como datos enlazados. Gordon Dunsire muestra la compatibilidad entre RDA y ONIX (Dunsire, 2007).

La Library of Congress tiene como uno de sus objetivos facilitar el acceso al conjunto de sus recursos y para ello trabaja en la elaboración de un único motor de búsqueda que pueda chequear los cerca de 20 millones de registros bibliográficos y de fondos a partir de METS (METS, 2011). METS responde a las siglas de Codificación y Transmisión Estándar de Metadatos y se aplica a partir de MODS meramente descriptivos, como MARCXML, EAD, TEI, KML; ALTO u ONIX; $y$, a partir de MODS, para artículos y sus registros vinculados; o para MODS que son registros de recursos (McCallum, 2007).

Rebecca Guenther (2011), por su parte, expone las realizaciones y posibilidades de los metadatos en el acceso a los recursos digitales de la Library of Congress. A tal fin, hay que trasformar los principios operativos para localizar y navegar entre los recursos digitales, en virtud del distinto nivel de explotación de los motores de búsqueda por parte de las diferentes categorías usuarios y las variaciones de conocimiento en el acceso y recuperación de la información deseada por los usuarios.

A ello contribuye, sin duda, el hecho de establecer listas maestras de elementos metadatos de uso general, el marcado de las listas mejores y la creación de perfiles de metadatos según qué iniciativas. Algunos de éstos ya están organizados (Metadata..., 2010).

La interfaz de programación de aplicaciones sirve para ofrecer los datos bibliográficos que deseamos vetar que sean descargados. OCLC experimenta un crecimiento importante en servicios API en su iniciativa Grid Services, facilitados por Developer Network, de OCLC (OCLC Developer Network, 2010). La recogida de datos se hace a partir del Open Archives Initiative Protocol for Metadata Harvesting (OAI-PMH), que se encuentra en múltiples aplicaciones software de bibliotecas que cuentan con repositorios. Funciona mediante el protocolo HTTP y su formato es el Dublin Core, MARCXML y MODS.

Este último ofrece una gran compatibilidad con las codificaciones de los distintos recursos y es menos detallado que MARC. El exponente de datos enlazados lo constituye la web Authorities and Vocabularies, de la Library of Congress, 
que ofrece una sistematización normalizada y concisa de los datos y la flexibilidad de búsqueda a partir de XML, que, por usar XQuery, facilita las búsquedas en entornos semejantes (SRU, $\mathrm{OAI}-\mathrm{PMH} \ldots$...); permite usar herramientas como RSS y otros alimentadores; y vincula los datos de autoridad de la Library of Congress. Más información al respecto se encuentra en la ALA Annual Conference 2010 (Riley, 2010).

En las dos últimas décadas, los desarrollos de la catalogación bibliográfica, en tanto que operación ocupada en presentar fiel y de manera representativa los datos descriptivos e indiciales de las creaciones intelectuales y artísticas, han estado motivados por el imperativo de superar las limitaciones de la automatización y por encontrar en la innovación digital soluciones a tales problemáticas.

La obra digital, en un medio digital y entre unos usuarios familiarizados con la tecnología digital, exige el replanteamiento de la operación de catalogar, en sus bases especulativas y en sus procedimientos mecánicos, a fin de adecuarse a los cambios técnicos y de la sociedad presentes.

\section{Gestores de las RDA}

\subsection{Asociaciones profesionales}

La institución profesional que mayores energías despliega en la adecuación de la catalogación bibliográfica a los retos de presente y futuro es, sin duda, la IFLA. Fundada en 1927, ha aportado a los avances teóricos y prácticos de esta disciplina un alto ritmo de progreso, bien diferente de la evolución generacional de la época anterior.

En 1936 se creó el Committee on Catalogue Rules, que centró sus líneas de trabajo en la consecución de unas reglas internacionales de catalogación. Desde entonces la trayectoria de unificación de las codificaciones de catalogación, la formalización de principios internacionales de catalogación, la consecución de los estándares de descripción y sobre el control y autoridad de los datos relativos a nombres, títulos y materias serán prioridades que se van haciendo realidad desde los años cincuenta del siglo XX.

Con su nombre original, o con la denominación de Section on Cataloguing, es la parte de la IFLA que se ocupa de los distintos aspectos que se integran en el Programa Internacional de Control Universal de la Bibliografía y MARC Internacional. Dada su trayectoria -y ante la necesidad de revisar el nivel de adecuación de las agencias bibliográfica nacionales a los estándares bibliográficos, las posibilidades ofrecidas por las bases de datos bibliográficas con tecnología web y el elevado coste derivado de los trabajos de catalogación-, fue la impulsora del Seminar on Bibliographic Records, de Estocolmo, en 1997 (Requisitos..., 2004, 28-31).

\subsection{Comisiones y grupos especiales}

Por la especialización y por la necesidad de cooperar con otras instituciones de profesionales, la IFLA y las agencias nacionales de bibliografía han formado grupos de expertos para el análisis y concreción final de las actuaciones conducentes a la culminación de sus proyectos y realizaciones.

En esta categoría de entidades gestoras de las RDA se encuentra el Joint Steering Committee for Revision of AACR. En la primavera de 2005 cambió parte de su denominación por "of Development of RDA", asumiendo el hecho de abandonar la reforma de las AACR2 y abordar el proyecto normativo de las RDA: resource description and access. Este nuevo marco de referencia para las codificaciones catalográficas comprende un amplio conjunto de directrices e instrucciones sobre los tipos de contenido y los medios de comunicación del universo bibliográfico, ya sea en su versión analógica o en edición digital (Joint..., 2009).

Tom Delsey publicó en 2002 Functional analysis of the MARC21 bibliographic and holdings formats, sobre la base de aplicar el modelo entidad-relación a las AACR2 y el MARC21. A él se debe, en 2005, el cambio de la orientación en la transformación de la reglamentación anglosajona hacia la RDA, publicada en junio de 2010 (RDA..., 2010).

De este modo, el Joint Steering Committee of Development of RDA es, de alguna forma, el responsable material último de la misma, en su versión de junio de 2010, y de su mantenimiento y revisiones permanentes. Para su difusión ha creado RDA Toolkit (RDA Toolkit, 2010) y The European RDA Interest Group (EURIG) (European..., 2010). The European RDA Interest Group (EURIG) está integrado por expertos catalogadores de distintas bibliotecas nacionales a fin de analizar el alcance, propiciar su implantación mundial y garantizar el mantenimiento de la actualización permanente de RDA.

Caroline Brazier (2010) muestra una esquematización sobre las instituciones presentes que hacen viable el futuro de RDA. Otras iniciativas han sido impulsadas por la OCLC (Ficción...,2 009), la National Library of Australia (Ayres, 
2005, p. 43-55), VTLS Inc, etc., sobre la base de los FRBR y, sobre todo, por la Library of Congress.

\subsection{Library of Congress}

El protagonismo de esta institución bibliotecaria a lo largo del siglo XX en la operación de catalogar es tan importante como el papel debido a la IFLA. En un análisis somero resulta difícil deslindar qué iniciativas de esta entidad bibliotecaria no tienen una transcendencia, de hecho, mundial. En 2000 se celebró el Bicentenario de su creación (Library..., 2000), y en este marco comenzó a revisarse, en profundidad, una tercera versión de las AACR. Desde 2005 los trabajos se recondujeron y el resultado ha sido la RDA (RDA..., 2010).

La Library of Congress ha desarrollado estándares propios y de alcance mundial, que afectan de manera nuclear a la catalogación tradicional, la automatizada y la de esta etapa caracterizada por la tecnología digital. Destacar sólo que es la impulsora de modelos de descripción de objetos metadatos (MODS), modelos de descripción de autoridad metadatos (MADS) y normas de codificación y transmisión de metadatos (METS). E, igualmente, significativo es el desarrollo del Equipo de Asesoramiento de Enriquecimiento Bibliográfico (BEAT), que ofrece informaciones suplementarias sobre autores, títulos o el índice de las obras. Estos modelos y herramientas se verán más adelante.

La RDA, presentada al público en junio de 2010, se inspira en los ICP (2009) y en los FRBR (1998) y en FRAD (2008). Ahora bien, es palmario que el modelo normativo de las AACR2 tiene un importante ascendiente en las reglamentaciones de las agencias bibliográficas nacionales de los países de Occidente. Así lo muestran los trabajos de comparación de reglas nacionales de catalogación, impulsados por la IFLA entre 2003 y 2007 y cuya relación completa consta en Principios de catalogación de IFLA... (2005).

\section{Caracterización de la RDA}

Las RDA constituyen el modelo de referencia para la descripción y el acceso a las obras en tecnología digital. Ésta condiciona la funcionalidad y la operatividad del prontuario bibliográfico, vehículo de comunicación del conocimiento y acceso al saber. Las RDA suponen la continuidad de la catalogación bibliográfica conforme al modelo catalográfico más reciente, frente a la catalogación automatizada, semimecánica, manual... característica de etapas anteriores.
Las RDA se han concebido como un instrumento de trabajo con tecnología web, cuya aplicación será gradual. Inicialmente, en la etapa de experimentación, su manejo se hace por acceso restringido de palabra clave. En la etapa de difusión dispondrá de una guía de catalogación y distintos niveles de uso en virtud del alcance y responsabilidad de las acciones.

Las RDA se integran por una terminología y una serie de conceptos que proceden de los modelos de entidad-relación definidos en FRBR y FRAD. De modo, que las directrices e instrucciones de las RDA se formulan a partir de la metodología asumida por tales modelos y consisten en priorizar el punto de vista del lector, investigador o del profesional en una serie de acciones.

Los FRBR sirven para: (1) encontrar las entidades que respondan a la secuencia de búsqueda planteada al catálogo y/o base de datos; (2) identificar la entidad, o entidades, entre los resultados ofrecidos como respuesta a la búsqueda hecha; (3) seleccionar aquella entidad que se ajusta a las exigencias específicas del lector o investigador entre las varias posibles, en dependencia de su necesidad concreta; y (4) adquirir por compra o préstamo u obtener el acceso en línea a la entidad encontrada, identificada y seleccionada.

Los FRAD se usan para: (1) encontrar la entidad, o entidades, según el criterio de búsqueda propuesto a partir de atributos o varios de éstos; (2) identificar una o varias entidades de las ofrecidas como resultado por el catálogo o la base de datos, ya sea con un fin informativo para la discriminación de entidades semejantes, ya sea para confirmar la forma del nombre empleada como punto de acceso controlado, ya sea para contextualizar una entidad, ya sea para singularizar conexiones entre las entidades; (3) aclarar, o justificar, el fundamento por el que la forma autorizada del nombre de la entidad es tal, y no otro; y (4) comprender la información ofrecida con su uso.

Las RDA dan especial valor al aspecto formal de los atributos de las entidades y a la serie de nexos que constituyen la trama indicial de cualquier registro bibliográfico y de autoridad, dado que constituyen la clave en el proceso de identificación de los elementos informativos y de recuperación de la información misma (Chris, 2010).

Una caracterización significativa de las RDA es su extensibilidad. En enero de 2010, todavía estaban pendientes de desarrollo instrucciones relativas a conceptos, objetos y eventos, $u$ otros, como ha indicado Barbara B. Tillet (2009). 
En realidad, se trata de conceptualizaciones de FRAD, que están a la espera de informes finales (Requisitos... de autoridad, 2009). Siendo esto así, fue razonable estimar que la versión de junio de 2010 de las RDA fuera la primera tanto por esta circunstancia como por las realidades de naturaleza eminentemente práctica que se deriven de su aplicación primera.

Las RDA aspiran a ser un código internacional de catalogación. Sus directrices e instrucciones refuerzan la descripción de los elementos informativos y la relación indicial que caracteriza al prontuario bibliográfico integrado por datos descriptivos y de autoridad ajustados a una codificación que facilita su transmisión hasta donde alcanza la tecnología digital. La globalización, que afecta a tantas actuaciones del hombre de hoy y a ciertos fenómenos de la naturaleza del presente, tiene su fiel traslado a la operación de catalogar por efecto de las TICs.

Las RDA se aplican a todos los documentos, analógicos y digitales, desde un marco de trabajo integrador, flexible y sujeto a cambios progresivos de orden técnico. Son receptivas a los principios, los estándares y otros modelos internacionales relativos a la catalogación bibliográfica. Todo ello hace que las instrucciones y directrices que integran las RDA constituyan una trama reglamentaria útil y compatible en archivos, museos, mundo editorial u otras organizaciones de información en las que la tecnología informática es determinante.

En las RDA pueden destacarse ocho rasgos básicos: (1) establecer los principios e instrucciones del registro de los datos; (2) permitir su aplicación a cualquiera de las comunidades del patrimonio cultural, ya sean bibliotecas, archivos, museos, repositorios digitales u otros; (3) ser un estándar polivalente, concebido y diseñado para obras y entornos digitales, y válido para los documentos analógicos; (4) servir en la creación de registros de datos que deben gestionarse por la tecnología al servicio de la comunicación del saber y creatividad; (5) expresar tales datos en secuencias de datos, caracterizados por referirse a un tipo particular de datos, definirse inequívocamente; (6) codificar tales datos en los esquemas actuales (MARC21, DC, MODS...) y de futuro a efectos de almacenar y de transmitir la descripción técnica de todo tipo de obras y de todo tipo de contenidos; (7) disponer de un procedimiento de aplicación múltiple, ya sea sobre el modelo de trabajo FRBR y FRAD, o como diagrama de entidad-relación, ya a partir de flujos de trabajo sobre procedimientos concretos; y (8) estar abierto a otros desarrollos conceptuales y formales, no incluidos en su primera versión, toda vez que aspira a tener un uso internacional.

En tiempos en los que los sistemas integrados de gestión bibliotecaria suponen un coste importante y en un ámbito profesional tan marcado por el peso de una formación especialmente empírica se ha hecho necesario agilizar la transición de la catalogación vigente a las RDA. A tal fin, se ha elaborado un documento que concreta los cambios que afectan a las AACR2 (Cambios..., 2009). En primer lugar, esto obedece al hecho de coincidir el ámbito de aplicación de tal normativa y el campo de experimentación y de ensayo de las RDA y, también, por el enorme influjo que conceptualmente y estructuralmente las AACR2 ejercen sobre el resto de reglamentaciones catalográficas nacionales.

\section{Conclusiones}

El concepto de universo bibliográfico, forjado por la IFLA entre 2003 y 2007, es un buen ejemplo de los cambios obrados en la expresión y comunicación de los conocimientos del presente. Los agentes responsables de crear y distribuir las obras de nuestros días participan de la creación y comercialización de tales obras en versión digital, dado que desde 2007 se constata el incremento paulatino de la demanda de productos digitales frente a los tradicionalmente impresos, en las sociedades más desarrolladas de Occidente.

La catalogación que se está experimentando en las principales bibliotecas y redes bibliotecarias de lengua inglesa presentan y comunican la información de los recursos digitales y analógicos a partir de la interoperabilidad de los distintos sistemas de codificación de datos bibliográficos, de emplear un mínimo de tales datos, o de su extensibilidad frente a nuevas realizaciones futuras.

Los objetivos de los responsables de la RDA y la tecnología digital han convergido en un mismo contexto espacial y temporal, de forma que la interrelación entre ambos ha resultado ser provechosa para el desarrollo sociocultural y económico de los países con mayor nivel de TICs.

La IFLA, a través de su Committee on Catalogue Rules, posee un alto nivel de responsabilidad en la generación de los marcos teóricos que sustentan las RDA y su realización material.

Asimismo, el Joint Steering Committee for Revision of AACR ha contribuido en la configuración de la primera versión de las RDA, en 2010. La nueva orientación dada por Tom Delsey al JSCR of AACR a partir de 2005 ha sido deter- 
minante en la elaboración de un código internacional de catalogación.

Las actuaciones del European RDA Interest Group son esenciales para que las RDA se implanten en los países continentales de Europa. Francia, Alemania, España, Italia... poseen importantes tradiciones nacionales de catalogación bibliográfica, frente al modelo de las AACR.

La Library of Congress ha promocionado la metamorfosis de las AACR2 en las RDA. También ha sido el marco de ensayo para los modeIos MODS, MADS, METS o MDC, que constituyen el marco tecnológico digital de referencia para hacer de la catalogación bibliográfica los metadatos. Tales modelos permiten asumir a los metadatos las funcionalidades de acceso y recuperación de la información, desempeñados tradicionalmente por la catalogación bibliográfica.

Las RDA se caracterizan por asumir la conceptualización y terminología de los FRBR (1997), los FRAD (2008) y los ICP (2009), siendo éstos los pilares sobre los que se asienta la actual teoría de la catalogación bibliográfica y las RDA (2010) la codificación internacional de la catalogación analógica y digital del presente.

\section{Referencias}

Abadal i Falgueras, Ernest (1994). El futuro de la edición ¿es electrónico?: tecnologías de creación y empleo de la información. // Jornadas Españolas de Documentación Automatizada. Los profesionales ante el reto del Siglo XXI: integración y calidad, Gijón: FESABID, 1994.

Alonso, J.; Cordón, J. A. (2010a). Libro electrónico y los DRM. Grupo ThinkEPI. http://www.thinkepi.net/2010/07 (2011-04-30).

Alonso, J.; Cordón, J. A., (2010b). El libro electrónico en el ecosistema de información. // Ciencias de la información. ISSN 1606-4925. 42, 2 (agosto 2010), 58-68. http://eprints.rclis.org/19030/1/Libro_electronico_(Cienca is_de_la_Informaci\%C3\%B3n).pdf (2011-04-19).

Ayres, Marie Louise (2005). Case studies in implementing Functional Requeriments for. for Bibliographic Records [FRBR]: AustLit and MusicAustralia". // ALJ: the Australian Library Journal. 54:1 (February 2005) 43-54. http://www.nla.gov.au/openpublish/index.php/nlasp/articl e/viewArticle/1225/1510 (2011-05-31).

Berners-Lee, Tim (2007). Metadata architecture. Documents, metadata, and links. Tim BL, January 1997. Last edit Date: 2009/08/27 21:38:08. http://www.w3.org/De signlssues/Metadata.html (2011-05-02).

Bibliographic Enrich Ment Advisory Team (2008). http://www.loc.gov/catdir/beat/ (2011-06-05).

Brazier, Caroline (2010). RDA future development: governance, participation and future change. // RDA in Europe: making it happen! (2010. Copenhague). http://www.slainte.org.uk/eurig/docs/RDA2010/BrazierE URIG2010.pdf (2011-04-20).

Cambios en las instrucciones de las AACR (2009). http://www.rda-jsc.org/docs/5sec7rev_spa.pdf (2011-0905).
Caplan, Priscilla (1995). You call it corn, we call it syntaxindependent metadata for documents-like objects. // The Public-acces computer systems review. ISSN 1063164X. 6:4 (1995) 19-23.

Chris, Oliver (2010). Introducing RDA: a guide to the basics. Chicago: American Library Association, 2010.

Cordón, J. A.; Alonso, J.; Martín, H. Los libros electrónicos: la tercera ola de la revolución digital (2010). // Anales de documentación. 13 (2010) 53-80. http://revistas .um.es/analesdoc/article/view/106991 (2011-05-10).

Cordón, J. A.; Gómez, R.; Alonso, J. (2010). Gutenberg 2.0: revolución de los libros electrónicos. Gijón: Trea, 2010.

Dunsire, Gordon (2007). Distinguishing content from carrier: the RDA/ONIX framework for resource categorization. Dlib magazine. ISSN 1082-9873. 13, 1-2 (2007). http://www.dlib.org/dlib/january07/dunsire/01dunsire.html (2011-05-31)

Encuesta sobre el libro digital: impacto de la digitalización en el catálogo, canales de distribución y venta y política de precios (2009). http://www.mcu.es/libro/docs/MC/CD/ Libro_Digital_2009.pdf (2011-04-30).

The European RDA Interest Group (EURIG) (2010). http://www.slainte.org.uk/eurig/index.htm (2011-03-30).

FictionFinder: a FRBR-based prototype for Fiction in WorldCat (2009). http://www.oclc.org/research/activities/fiction finder/default.htm (2011-05-24).

Gradmann, Stefan (1998). Catalogación versus metadata: vino viejo en odres nuevos?. 64th IFLA General Conference, 16-21 August Amsterdam. http://archive.ifla. org/IV/ifla64/007-126s.htm (2011-06-04).

Guenther, Rebecca (2011). Using metadata standard in digital libraries: implementing MTES, MODS, PREMIS and MIX: introduction. http://cc.bingj.com/cache.aspx? $q=$ Rebecca+Guenther+MODS\&d $=4623815889652905 \&$ $\mathrm{mkt}=\mathrm{es}-\mathrm{ES} \&$ setlang $=\mathrm{es}-\mathrm{ES} \& \mathrm{w}=\mathrm{c} 6760 \mathrm{ae} 5,83 \mathrm{e} 84590$ (2011-05-30)

Hsieh-Yee, Ingrid (1997). Modificaciones a las reglas de catalogación y la infraestructura de OCLC para una organización efectiva de los recursos de Internet. http://cuib.unam.mx/ felipe/cat_Internet/modificaciones. $\mathrm{htm}$ (2011-05-23).

Joint Steering Committee for Revision of AACR (2009). http://www.rda-jsc.org/docs.html (2011-04-04).

Lagoze, Karl (1997). From static to dynamic surrogates: resource dicovery in the Digital Age. // D-lib magazine. ISSN 1082-9873. (June 1997). http://www.dlib.org/delib/ june97/06lagoze.html (2011-05-22).

Library of Congress Bicenntenial http://www.loc.gov/bicentennial/ (2011-04-01).

(2000).

El libro electrónico (2010). Por el Grupo de Trabajo de EI Libro Electrónico. http://www.mcu.es/libro/docs/MC/Ob servatorio/pdf/LIBRO_ELECTRONICO_2010.pdf (201104-25).

McCallum, Sally (2007). MARC futures. http://www.mcdu.unt.edu/wp-content/ALA2007McCallum MARCFutures.pdf (2011-05-10).

Méndez Rodríguez, Eva María (2002). Metadatos y recuperación de la información: estándares, problemas y aplicabilidad en bibliotecas digitales. Gijón: Trea, 2002.

Méndez Rodríguez, Eva María (2010). Tendencias en recuperación de información: principios y retos para una nueva década de datos enlazados. // Anuario ThinkEPI. (2010) 1 .

Metadata for Digital Content (MDC): developing Institutionwide policies and standards at the Library of Congress (2010). http://www.loc.gov/standards/mdc/ (2011-06-06).

METS: Metadata Encoding \& Transmission Standard (2011). http://www.loc.gov/standards/mets/ (2011-04-30). 
OCLC Developer Network (2010). http://www.oclc.org/debe loper/ (2011-06-05).

The Open Archives Initiative Protocol for Metadata Harvesting (2008). http://www.openarchives.org/OAl/2.0.bac kup.2008-12-07/openarchivesprotocol.2002-08-06.htm (2011-06-05)

Pasch, Grete (2001). Los metadatos y el reto de las tradiciones bibliográficas. http://www.ucol.mx./interfaces/ \%20interfaces2001/archivos/gretepasch.pdf (2011-0430).

Patiño, Bruno (2009). Informe sobre el libro digital en Francia. // Revista pensar el libro. 6 (junio 2009) 4-5. http://www.cerlalc.org/revista_junio/revista_pensarellibro _menu.htm (2011-05-02).

RDA: resource description and access (2010). http://www.rda-jsc.org/rda.html (2011-05-10)

RDA Toolkit (2010). En: http://www.rdatoolkit.org/ (2011-0330).

Requisitos funcionales de los registros bibliográficos. Informe final (2004). Madrid: Ministerio de Cultura, Secretaría General Técnica, 2004.

Requisitos funcionales de los datos de autoridad (FRAD): un modelo conceptual. Informe final, diciembre de 2008 (2009). Ed. by Glenn E. Patonn. Ed española. http://www.bne.es/es/Servicios/NormasEstandares/Docs /FRAD_espaxol.pdf (2011-09-05).

Riley, Jenn (2010). MODS and MADS: current implementations and future directions. http://presentations.ala.org/ index.php?title=Sunday,_June_27\#MODS_and MADS: Current Implementations and Future Directions (2011-06-06).

Rusch-Feja, Diann D (1998). Metadata: standards for retrieving WWW documents (and other digitized and nondigitized resources). // Library and information services in astronomy, III ASP Conference Series. 153 (1998). http://www.stsci.edu/stsci/meetings/lisa3/reprints/ruschfe jad.pdf (2011-06-04).

Tennant, Roy (2009). 21th Century description and Access. // BID: textos universitaris de biblioteconomía i documentació. ISSN 1575-5886. 2 (2009). http://www.ub.edu/bid/22/tennant2.htm (2011-06-05).

Tillet, B. B.; Gömpel, R.; Oehlschläger, S. (Eds.) (2005). Principios de catalogación de IFLA: pasos hacia un código internacional de catalogación. Madrid: Ministerio de Cultura, 2005. http://www.ifla.org/files/cataloguing/icc/ime-icc-1-es.pdf (2011-05-25).

Tillet, Barbara B. (2009). RDA: antecedentes y aspectos de su implementación. http://www.loc.gov/catdir/cpso/ RDA/RDAantecedentes_instructor.pdf (2011-06-05).

Torres Pombert, Ania (2006). ¿Catalogación en el entorno digital?: una breve aproximación a los metadatos. I/ Acimed. ISSN 1530-2880. 14, 5 (2006). http://www .bvs.sld.cu/revistas/aci/vol14 5 06/aci09506.htm (201106-03).

Van Nuys, C.; Albertsen, K.; Pedersen, L.; Stenstad, A. (2004). El Proyecto Paradigma y su búsqueda de soluciones para metadatos y servicios a usuarios. // World Library and Information Congress: 70th IFLA General Conference and Council, 22-27 August 2004, Buenos Aires. http://ifla.queenslibrary.org/IV/ifla70/papers/009s_t rans_Nuys.pdf (2011-05-31).

Weibel, S.; Hekela, J. (1998). DC-5: the Helsinki Metadata Workshop: a report of the Workshop and Subsequent Developments: oficial report of the Helsinki DC Meeting. // D-lib magazine. ISSN 1082-9873. February 1998. http://www.dlib.org/dlib/febuary98/02weibel.html (201105-22).

Enviado: 2011-09-07. Versión corregida: 2011-09-07. Aceptado: 2011-09-23. 
